\title{
Aspergillus flavus grown in peptone as the carbon source exhibits spore density- and peptone concentration-dependent aflatoxin biosynthesis
}

Shijuan Yan ${ }^{1,2}$, Yating Liang ${ }^{1,3}$, Jindan Zhang ${ }^{2}$ and Chun-Ming Liu ${ }^{2 *}$

\begin{abstract}
Background: Aflatoxins (AFs) are highly carcinogenic compounds produced by Aspergillus species in seeds with high lipid and protein contents. It has been known for over 30 years that peptone is not conducive for AF productions, although reasons for this remain unknown.

Results: In this study, we showed that when Aspergillus flavus was grown in peptone-containing media, higher initial spore densities inhibited AF biosynthesis, but promoted mycelial growth; while in glucose-containing media, more AFs were produced when initial spore densities were increased. This phenomenon was also observed in other AF-producing strains including A. parasiticus and A. nomius. Higher peptone concentrations led to inhibited AF production, even in culture with a low spore density. High peptone concentrations did however promote mycelial growth. Spent medium experiments showed that the inhibited AF production in peptone media was regulated in a cell-autonomous manner. mRNA expression analyses showed that both regulatory and AF biosynthesis genes were repressed in mycelia cultured with high initial spore densities. Metabolomic studies revealed that, in addition to inhibited AF biosynthesis, mycelia grown in peptone media with a high initial spore density showed suppressed fatty acid biosynthesis, reduced tricarboxylic acid (TCA) cycle intermediates, and increased pentose phosphate pathway products. Additions of TCA cycle intermediates had no effect on AF biosynthesis, suggesting the inhibited AF biosynthesis was not caused by depleted TCA cycle intermediates.

Conclusions: We here demonstrate that Aspergillus species grown in media with peptone as the sole carbon source are able to sense their own population densities and peptone concentrations to switch between rapid growth and AF production. This switching ability may offer Aspergillus species a competition advantage in natural ecosystems, producing AFs only when self-population is low and food is scarce.
\end{abstract}

Keywords: A. flavus, A. parasiticus, A. nomius, Peptone, Aflatoxin biosynthesis, Density effect

\section{Background}

Aflatoxins (AFs) are a group of polyketide metabolites produced by several toxigenic species of Aspergillus such as $A$. flavus and $A$. parasiticus after infections of seeds with high protein and lipid contents, e.g. peanut, corn and walnut [1-3]. AFs are toxic and carcinogenic, posing serious threats to both animal and human health [4]. Extensive studies carried out in A. flavus and A. parasiticus lead to the identification of a $70 \mathrm{~kb}$ DNA cluster consisting two specific transcriptional regulators (aflR and aflS),

\footnotetext{
* Correspondence: cmliu@ibcas.ac.cn

${ }^{2}$ Key Laboratory of Plant Molecular Physiology, Institute of Botany, Chinese Academy of Sciences, 20 Nanxincun, Fragrant Hill, Beijing, 100093, China Full list of author information is available at the end of the article
}

and 26 co-regulated downstream metabolic genes in the AF biosynthetic pathway [5-8]. Expressions of aflR and aflS are further regulated by global regulators such as the CreA transcription factor and the VelB/VeA/LaeA complex, and possibly by a cell surface-localized Gprotein coupled receptor complex $[2,9,10]$.

Various nutritional and environmental factors including carbon sources [11], nitrate [12], light [13], temperature $[14,15], \mathrm{pH}[14,16]$, and oxygen availability [17-19] affect $\mathrm{AF}$ productions and expressions of AF biosynthesisrelated genes $[9,20,21]$. It has been known for a long time that sugars and related carbohydrates support both fungal growth and AF production. However, peptone, a mixture of protein degradation products, is a preferred carbon

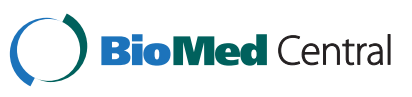


source for fungal growth, but not for AF production [11,22-25]. Many studies have been carried out to elucidate how various carbon sources affect AF biosynthesis. Transition from peptone mineral salts (PMS) medium to glucose mineral salts (GMS) medium leads to AF biosynthesis, a process requiring de novo transcription and translation [24]. Comparisons of a large collection of carbon sources reveal that sugars that are normally oxidized through the hexose monophosphate or glycolytic pathway such as glucose, raffinose and mannose are efficient carbon sources for AF productions [23], while lactose and most amino acids excluding aspartate are considered to be unsuitable carbon sources for AF production $[11,26]$. AFs are usually produced in parallel with fatty acid biosynthesis following the rapid growth and sugar utilization phase, as common precursors such as acetyl-CoA and malonyl-CoA derived from glucose catabolism are utilized in both pathways [18]. As many carbohydrates are able to induce AF production, Abdollahi and Buchanan (1981) believe that utilization of readily metabolized carbohydrates may result in elevated energy status which in turn induces AF biosynthesis [23]. Wiseman and Buchanan (1987) note that, although mycelia grow well in media with low concentrations of suitable sugars, AFs are produced only when sugar concentrations are higher than $0.1 \mathrm{M}$, and in which reduced mycelial growth and inhibited TCA cycle activity are observed [27]. Addition of TCA cycle intermediates inhibits AF production, suggesting that glucose may regulate $\mathrm{AF}$ productions through inhibition of the TCA cycle $[25,26]$. Recent studies have revealed cell density-dependent sclerotium formation and AF production in media with glucose and sorbitol as the carbohydrate sources, which is regulated through non-cell autonomous factors [28,29].

In nature, seeds with high protein and lipid content, such as peanut and cotton, are more susceptible to high AF production than starchy seeds like rice and sorghum [1]. It has also been shown in maize that mycelial growth and AF production occur primarily in the embryo and the aleurone layer where mainly storage proteins and lipids are accumulated [30,31]. Removal of oil from ground cotton seeds greatly enhances AF production, suggesting that lipids are not essential for optimal AF biosynthesis [32]. Fatty acids may stimulate or inhibit AF production through the presence of various oxidationderived oxilipins [33-36]. The influence of protein and peptone on AF biosynthesis remains largely unknown.

In this study we investigated how AF production by Aspergillus was influenced when peptone was used as the sole carbon source. Contrary to expectations, we observed spore density- and peptone concentrationdependent AF production with peptone as the sole carbon source. AFs were only produced in the PMS medium when initial spore densities were $10^{4}$ spores $/ \mathrm{ml}$ or lower. In contrast, mycelia cultured in the PMS medium with higher initial spore densities or with increased peptone concentrations grew rapidly but without AF production. Spent media experiments showed that no inhibitory factors were released into the culture media. Metabolomic analyses revealed that, in addition to inhibited AF biosynthesis, mycelia grown in peptone media with high initial spore densities showed enhanced sugar utilization and repressed lipid biosynthetic metabolism.

\section{Results}

\section{Spore density-dependent AF production in PMS media}

PMS has long been considered to be a non-conducive medium for AF production in both A. flavus and $A$. parasiticus [23-25]. To investigate the mechanism underlying peptone's influence on AF biosynthesis, the well-studied A. flavus A3.2890 [37-39] from the China General Microbiological Culture Collection Center (CGMCC) was used to conduct our experiments. It was indeed the case that $A$. flavus did not produce AFs when cultured at the commonly employed initial spore density of $10^{5}$ or $10^{6}$ spores $/ \mathrm{ml}$. However, when various spore densities of A. flavus were tested to initiate cultures, a density-dependent AF production was observed. When the initial spore density was gradually decreased, increasing amounts of AFs were detected in media after 3day culture, as shown by thin-layer chromatography (TLC) and high pressure liquid chromatography (HPLC) analyses (Figure 1B \& D). At $10^{1}$ spores $/ \mathrm{ml}$, the amount of AFs produced was significantly lower, comparable to that of the $10^{4}$ spores $/ \mathrm{ml}$ culture. The maximal AF production was observed in the PMS medium inoculated with $10^{2}$ spores $/ \mathrm{ml}$. This differs from GMS cultures, where increasing amounts of AFs were produced when initial spore densities were increased from $10^{1}$ to $10^{6}$ spores/ml (Figure 1A \& C). We also observed that in GMS media, AFB1 was the major toxin (Figure 1C), while in PMS media, AFG1 was the primary toxin produced (Figure 1D). These data suggest that AF biosynthesis is regulated differentially in these two media.

Since most $A$. flavus strains produce only AFB1 [40-42], we examined if the A3.2890 strain used was indeed A. flavus. By using the protocol developed by Henry et al (2000) [43], fragments of the internal transcribed spacer (ITS) region of $r R N A, \beta$-Tubulin and Calmodulin genes from the A. flavus A3.2890 strain were amplified and sequenced, and then compared with corresponding sequences in the Genbank, and confirmed that A3.2890 is indeed A. flavus (see Additional files 1, 2, 3 and 4). It is very likely that the strain we used belongs to the type IV A. flavus, which produces both AFBs and AFGs, as reported recently [44]. 

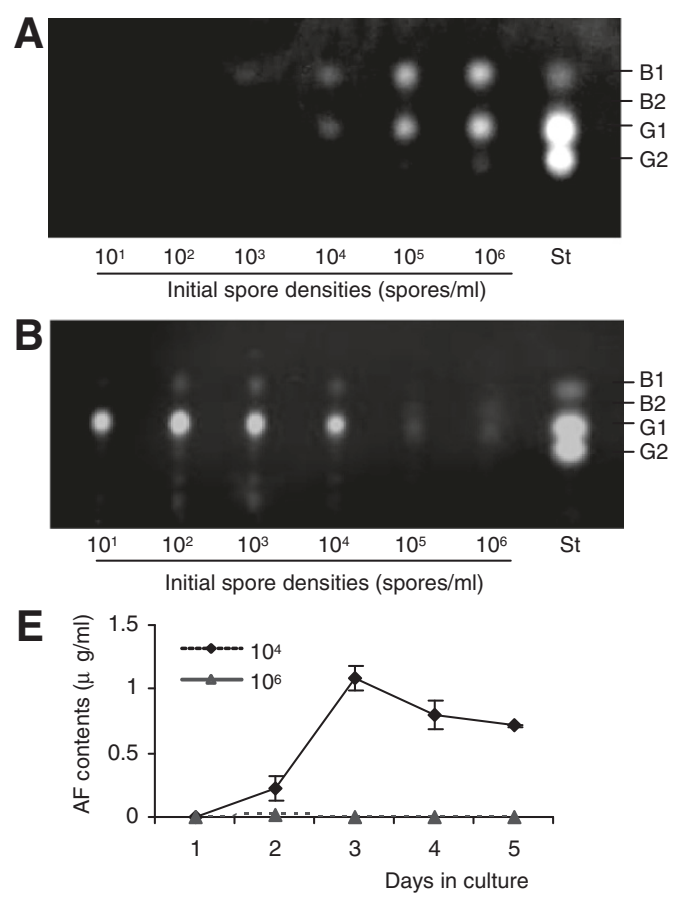
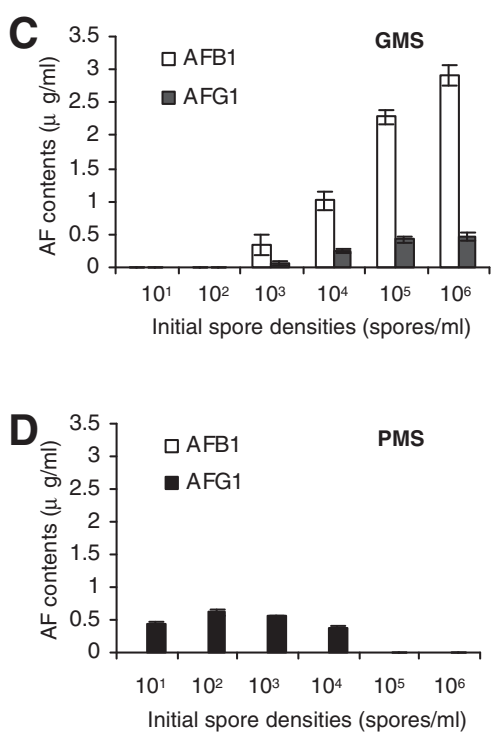

Figure 1 Spore density-dependent AF productions in A. flavus in PMS media. (A, B), TLC analyses of AF productions by A. flavus A3.2890 cultured in GMS (A) or PMS (B) media for 3 days with initial spore densities of $10^{1}, 10^{2}, 10^{3}, 10^{4}, 10^{5}$ and $10^{6}$ spores $/ \mathrm{ml}$. Ten $\mu \mathrm{l}$ AF extracts were loaded in (A), and $50 \mu \mathrm{l}$ in (B). St: AF standards. (C, D) HPLC analyses of AFs produced by A. flavus A3.2890 cultured in GMS (C) or PMS (D) media for 3 days, with the initial spore densities of $10^{1}, 10^{2}, 10^{3}, 10^{4}, 10^{5}$ and $10^{6}$ spores $/ \mathrm{ml}$. Note in GMS media both AFB1 and AFG1 were produced, while in PMS media mainly AFG1 was produced. (E) The time course of AFG1 productions in PMS media during 5-day cultures, with initial spore densities of $10^{6}$ (dotted line) or $10^{4}$ (solid line) spores $/ \mathrm{ml}$. All results were the mean \pm SD of 3 measurements from mixed three

independent samples.

\section{The time course of AF production}

To assess the production and possible degradation of AFs during the cultural period with various initial spore densities, we examined AFG1 contents in the PMS medium during a five-day culture period, with $10^{6}$ or $10^{4}$ spores $/ \mathrm{ml}$. We observed that, in the culture initiated with $10^{4}$ spores $/ \mathrm{ml}$, a significant amount of AFG1 was detected on the day two, reached the maximum level on the day three, and subsequently decreased gradually. In contrast, almost no AFs were detected in the culture initiated with $10^{6}$ spores $/ \mathrm{ml}$ during the entire five-day culture period (Figure 1E). It has been shown previously that peptone from different suppliers may induce different enzyme activities in Candida albicans [45]. The peptone initially used in this study was purchased from Beijing Aoboxing Biotech. To ensure the result observed is a general phenomenon, peptone from Sigma and Shuangxuan Microbe Culture Medium Products Factory was tested, and same results were observed (see Additional file 5).

To examine if cultures with high initial spore densities lead to a similar AF accumulation in mycelia, we used the TLC method to analyze AF contents in mycelia cultured for three days in either PMS or GMS media, with $10^{4}$ or $10^{6}$ spores $/ \mathrm{ml}$. The results showed greatly reduced AF content in mycelia in culture initiated with $10^{6}$ spores $/ \mathrm{ml}$, similar to the AF content of the media. In contrast, increased AF production was observed in mycelia cultured in GMS media with $10^{6}$ spores $/ \mathrm{ml}$, as compared to that with $10^{4}$ spores $/ \mathrm{ml}$ (see Additional file 6).

\section{High initial spore density in PMS media led to rapid mycelial growth}

To exclude the possibility that the reduced AF production in PMS media initiated with high initial spore densities was caused by inhibited fungal growth, mycelium dry weights were determined during a five-day culture period. A. flavus cultured in GMS media with an initial density of $10^{4}$ or $10^{6}$ spores $/ \mathrm{ml}$ showed a similar growth curve, with a continuous increase in dry weight during the five-day incubation. Higher initial spore density led to slightly faster mycelial growth, and an increased mycelium dry weight (Figure 2A). A. flavus cultured with $10^{4}$ spores/ml in PMS media showed a similar growth curve to that in GMS media with the same spore density (Figure 2B). However, a much sharper exponential growth phase was observed in the first two days in PMS 

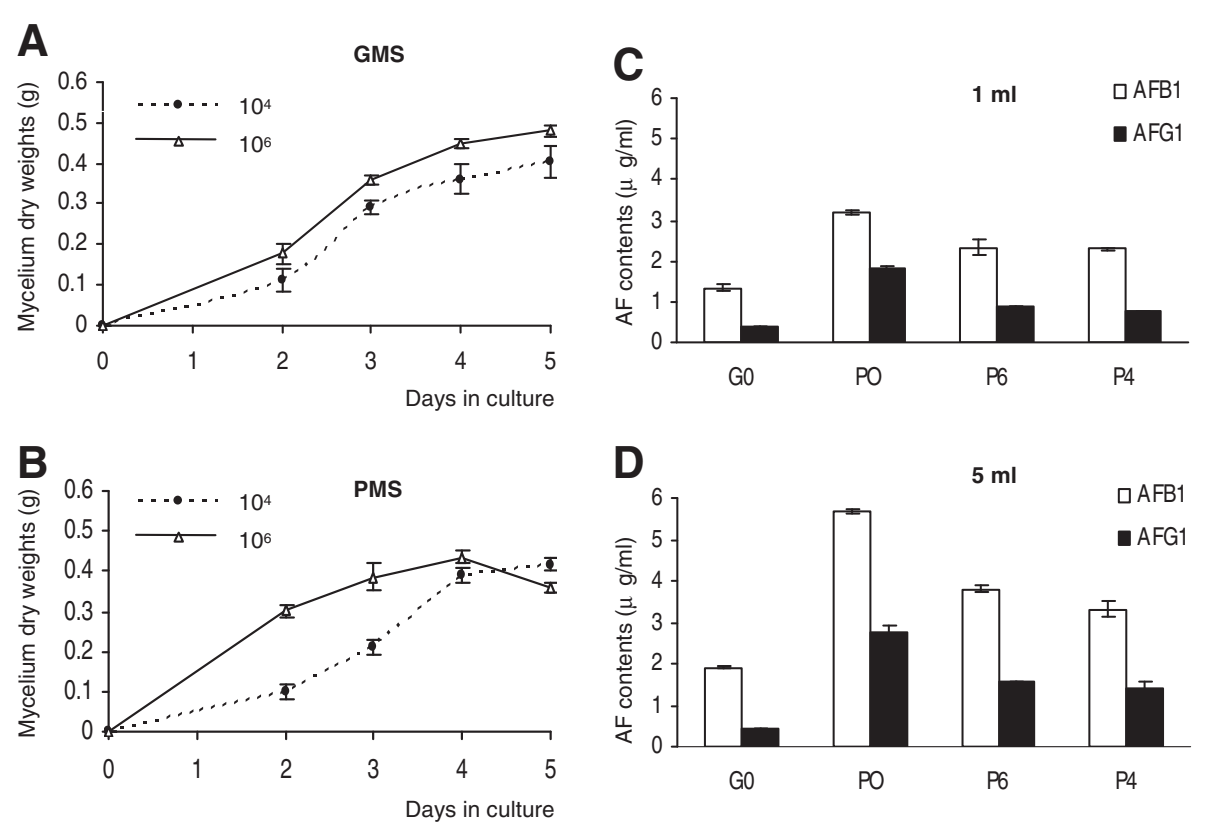

Figure 2 Mycelial growth and AF production of A. flavus cultured with different initial spore densities. (A, B) Mycelial growth curves of $A$. flavus A3.2890 in $50 \mathrm{ml}$ GMS (A) or PMS (B) media initiated with $10^{4}$ (dotted line) or $10^{6}$ spores $/ \mathrm{ml}$ (solid line). The mycelium dry weights were measured during a period of 5 days. (C, D) Effects of PMS spent media on AF productions. (C) One ml fresh GMS (G0) or PMS (P0) media, or spent media (P4 and P6) were added to GMS media inoculated with $10^{6}$ spores/ml. (D) Five $\mathrm{ml}$ fresh GMS (G0) or PMS (P0), or spent media (P4 and P6) were added to GMS media inoculated with $10^{6}$ spores $/ \mathrm{ml}$. AF contents were measured after cultured at $28^{\circ} \mathrm{C}$ for 3 days. The spent media were prepared from 3-day PMS cultures with the initial spore densities of $10^{4}(\mathrm{P} 4)$ or $10^{6}(\mathrm{P} 6) \mathrm{spores} / \mathrm{ml}$. All data were the mean $\pm \mathrm{SD}$ of $3 \mathrm{HPLC}$ measurements from mixed three independent samples.

culture initiated with $10^{6}$ spores/ml (Figure $2 \mathrm{~B}$ ). The mycelium dry weight reached the maximum level on the $4^{\text {th }}$ day and decreased significantly afterwards, suggesting no inhibition of growth in the high density PMS culture. Instead, A. flavus cultured in PMS media with a high initial spore density grew faster and degenerated earlier (Figure 2B).

\section{No inhibitory factor was released from the high density culture into the media}

We examined whether inhibitory factors were released into the media by A. flavus grown in PMS media with high initial spore densities. The experiment was performed by adding filter-sterilized spent media collected from 3-day cultures with $10^{4}$ or $10^{6}$ spores $/ \mathrm{ml}$ to fresh GMS media inoculated with $10^{6}$ spores $/ \mathrm{ml}$. Filtersterilized fresh PMS or GMS media were used as controls. The addition of $1 \mathrm{ml}$ fresh PMS medium (P0) to GMS cultures enhanced production of both AFB1 and AFG1, as compared to the addition of fresh GMS medium (G0) (Figure 2C), which is in agreement with a previous report [46]. As showed in Figure 2C, addition of $1 \mathrm{ml}$ spent media from both high (without AF production) and low (with AF production) density cultures to the GMS culture promoted AF production. No significant difference in AF production was observed in the high density culture. The experiment was extended further to add $5 \mathrm{ml}$ spent media from high (P6) and low (P4) density cultures. If inhibiting factors were present in the spent media, we would expect to see reduced AF productions when compared to addition of $1 \mathrm{ml}$ spent media. However, we observed that more AFs were produced in both P4 and P6 cultures, and no significant difference was observed between P4 and P6 samples (Figure 2D). Lower levels of AFs were produced in cultures with spent PMS media than those with fresh PMS media (Figure $2 \mathrm{C} \& \mathrm{D}$ ), which could be explained by nutrient consumption during the three-day incubations. These data together show that there seems to be no inhibitory factor released from the high density culture to the media.

\section{Increased peptone concentrations inhibited AF production}

To examine if the lack of AF production in PMS media with high initial spore densities is caused by rapid mycelial growth, and consequent depletion of nutrients, the peptone concentration in media from the original 5\% was increased to $15 \%$ to see if AF production could be restored. We observed, conversely, that mycelia cultured with increased peptone concentration showed greatly reduced AF production, regardless of initial spore 
densities $\left(10^{4}\right.$ or $10^{6}$ spores $/ \mathrm{ml}$ ) (Figure $3 \mathrm{~A}, \mathrm{P} 4+$ and $\mathrm{P} 6+)$. We then examined the mycelial growth in media with 5\%,10\% and $15 \%$ peptone, and observed increased mycelium dry weights when the peptone concentrations were increased (Figure 3B), suggesting that high concentrations of peptone promoted mycelial growth and at the same time inhibited AF biosynthesis. For each of the peptone concentrations, it was observed that cultures with higher initial spore densities showed an increase in mycelial growth. Taken together, these studies revealed that high concentrations of peptone promoted mycelial growths but inhibited AF production, suggesting that $A$. flavus grown in the peptone medium is able to sense the peptone concentrations and is able to shift between fast growth and $\mathrm{AF}$ production.

It has been reported previously that carbon sources affect the $\mathrm{pH}$ of culture media $[14,16]$. If AF production in media correlates with the $\mathrm{pH}$ changes was examined during incubation. We found that, as reported by Buchanan and Lewis (1984) [25], the $\mathrm{pH}$ of cultures in GMS media was decreased (Figure 3C, G4 and G6), while pH of cultures in PMS media was increased during the 55hr cultures (Figure 3C, P4, and P6). Higher initial spore density led to faster acidification or alkalization of the GMS and PMS media during the cultures, respectively (Figure 3C). Interestingly, we observed that when the peptone concentration was increased to $15 \%$, the $\mathrm{pH}$ of the media increased in the same way as the $5 \%$ peptone media (Figure 3C, $\mathrm{P} 4+$ and $\mathrm{P} 6+$ ). AF production was inhibited in the medium with $15 \%$ peptone, while AF production was active in the medium with $5 \%$ peptone, which suggests no direct connection between AF production and $\mathrm{pH}$ changes in our incubation system.

\section{High initial spore densities in PMS media repressed the expression of AF biosynthesis-related genes}

To further study how initial spore densities affect AF production in A. flavus, expression of AF biosynthesisrelated genes was examined by quantitative reverse transcription PCR (qRT-PCR) in mycelia initiated with $10^{4}$ or $10^{6}$ spores $/ \mathrm{ml}$ for two days. We observed that the expression levels of two transcriptional regulators (alfR and alfS), and three AF biosynthesis genes (aflO, cypA and $\operatorname{ord} A$ ) from the AF biosynthesis gene cluster were substantially lower in mycelia initiated with $10^{6}$ spores/ $\mathrm{ml}$, as compared to those initiated with $10^{4}$ spores $/ \mathrm{ml}$ (Figure 4A). The differences were even more pronounced on the day three (Figure 4B), suggesting transcriptional activation of AF biosynthesis in cultures initiated with the low spore density. We noted that nadA, which is involved in the conversion of AFG1 [47], showed increased expression in the culture initiated with
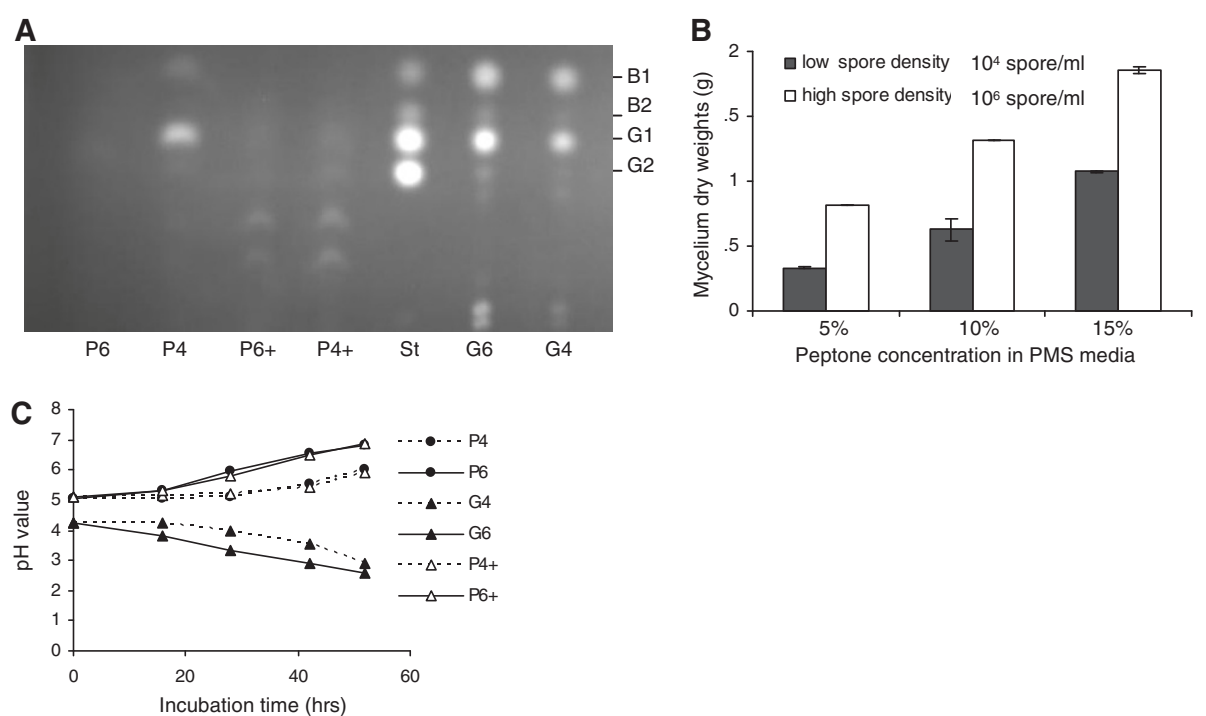

Figure 3 A. flavus grown in PMS and GMS media responded differently to the initial spore densities. (A) Higher concentrations of peptone inhibited AF productions in A. flavus A3.2890. P4, PMS media with the initial spore density of $10^{4}$ spores/ml; P6, PMS media with the initial spore density of $10^{6}$ spores $/ \mathrm{ml}$; G4, cultured in GMS media with the initial spore density of $10^{4}$ spores/ml; G6, cultured in GMS media with the initial spore density of $10^{6}$ spores/ml; P4+, PMS media with $15 \%$ peptone, cultured with the initial spore density of $10^{4}$ spores $/ \mathrm{ml}$; P6+, PMS media with 15\% peptone, cultured with the initial spore density of $10^{6} \mathrm{spores} / \mathrm{ml}$, St, AF standard. (B) Higher concentrations of peptone promoted mycelial growths. The total mycelium dry weights were measured after a 3-day culture, with initial spore densities of $10^{4}$ or $10^{6}$ spores/ml. (C) No direct correlations between AF productions and pH changes. In GMS media the pH was gradually decreased during the 55-hr culture, where a higher initial spore density led to faster acidification of the medium. In PMS media the pH was increased during culture, where a higher initial spore density led to rapid alkalization of the medium. Note that increased peptone concentrations did not cause a significant change in the $\mathrm{pH}$ of PMS media (P6 and P6+). 


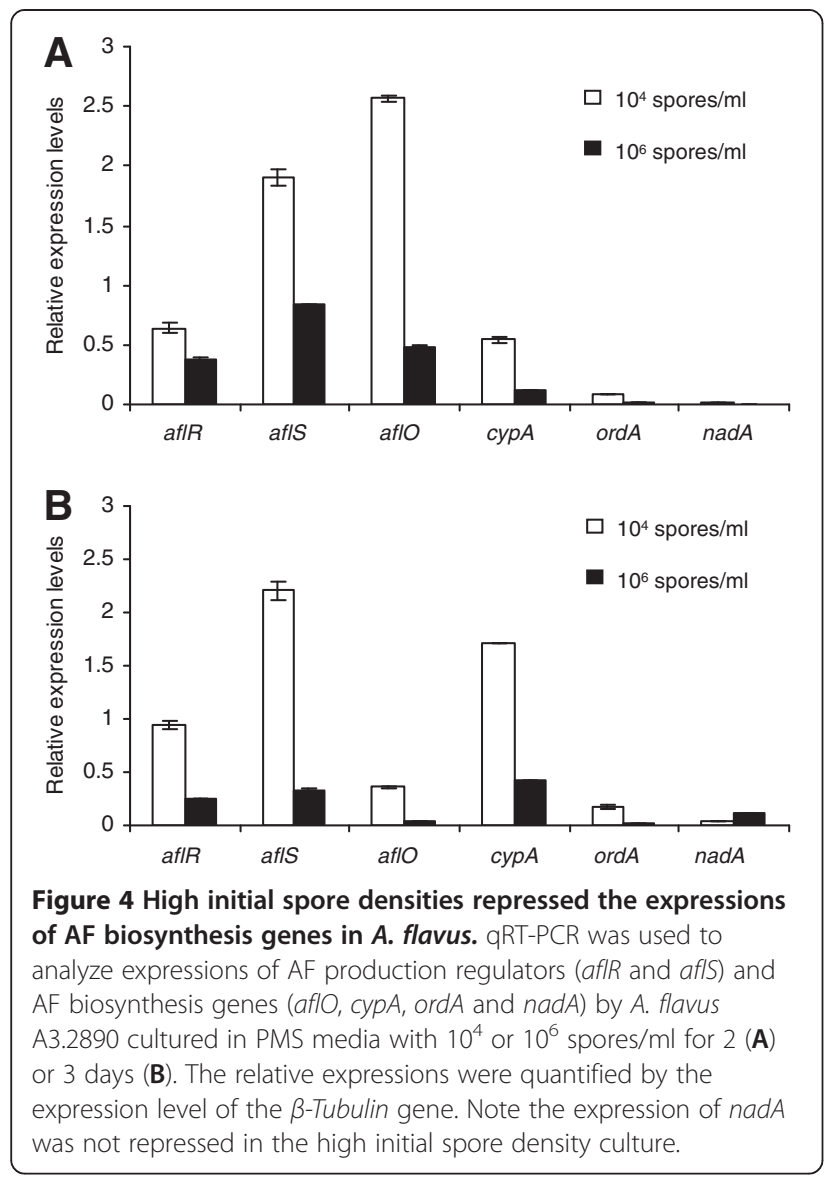

$10^{6}$ spores $/ \mathrm{ml}$, compared to those initiated with $10^{4}$ spores $/ \mathrm{ml}$ on the day three (Figure $4 \mathrm{~B}$ ).

\section{The density effect was present in most Aspergillus strains tested}

To elucidate if the density effect is a general phenomenon in AF-producing strains, we obtained $A$. flavus NRRL 3357, A. parasiticus NRRL 2999 and A. nomius NRRL 13137 from the Agricultural Research Service (ARS) culture collection in United States Department of Agriculture (USDA), and performed experiments in parallel with A. flavus A3.2890. Fresh spore suspensions were prepared in the same way as for A. flavus A3.2890, and inoculated in PMS or GMS liquid media with initial spore densities from $10^{2}$ spores $/ \mathrm{ml}$ to $10^{6}$ spores $/ \mathrm{ml}$. After three-day cultures, AFs were extracted from media and analyzed by TLC. As shown in Figure 5, in GMS media, all strains showed increased AF productions when initial spore densities were increased from $10^{2}$ to $10^{6}$ spores $/ \mathrm{ml}$, excluding A. flavus NRRL 3357. As reported previously, only AFB1 and AFB2 were produced by $A$. flavus NRRL 3357 [48], while for all other strains AFB1 and AFG1 were the major AFs produced.
In PMS media, similar to what was showed above in $A$. flavus A3.2890, we observed that high initial spore densities inhibited AF biosynthesis in A. parasiticus NRRL 2999 and A. nomius NRRL 13137, especially when initial spore densities were $10^{5}$ spores $/ \mathrm{ml}$ or higher (Figure 5). However, no AF biosynthesis was observed in A. flavus NRRL 3357 in PMS media, no matter the initial spore density. It seems somehow the A. flavus NRRL 3357 strain has lost the density sensing machinery in evolution.

\section{Mycelia grown in PMS media with high initial spore densities showed reduced TCA cycle intermediates and fatty acid accumulations, but enhanced PP pathway products}

To determine metabolic differences in A. flavus grown in PMS media with high or low initial spore densities, metabolites in mycelia cultured for 2, 3, 4 and 5 days were analyzed by gas chromatography time-of-flight mass spectrometry (GC-Tof-MS) using methods described previously [49,50]. Multi-variate analyses showed that mycelia inoculated with $10^{4}$ spores $/ \mathrm{ml}$ clustered separately from mycelia inoculated with $10^{6}$ spores/ml, suggesting evident metabolic differences between these two cultures (Figure 6A \& B). Striking differences in levels were observed in 24 metabolites on the $3^{\text {rd }}$ day (Figure $6 \mathrm{C} \& \mathrm{D}$, and Table 1). In PMS cultures initiated with $10^{6}$ spores $/ \mathrm{ml}$, a condition without AF production, the level of three TCA cycle intermediates, namely malic acid, fumaric acid and succinic acid, accumulated significantly less than those in cultures initiated with $10^{4}$ spores $/ \mathrm{ml}$ This suggests that the TCA cycle was more active in the high density culture. Similarly, levels of four fatty acids, palmitic acid, stearic acid, oleic acid and linoleic acid, were reduced in cultures initiated with the high spore density (Table 1), indicating that fatty acid biosynthesis was generally inhibited in the high density culture. In contrast, many sugar metabolites including ribitol, glucopyranoside, gluconolactone-6-P, glycerol, butanediamine, ethylamine and galactose, were accumulated more in the high density cultures (Table 1), suggesting that the PP pathway was active. In addition, nucleotides and compounds involved in amino acid metabolism were less abundant in cultures initiated with the high spore density (Table 1), which may be the consequence of the rapid mycelial growth.

\section{Addition of TCA cycle intermediates did not affect AF biosynthesis}

To test if reduced TCA cycle intermediates in mycelia are the primary cause of reduced AF biosynthesis in the high initial spore density culture, malic acid, fumaric acid and succinic acid were added to the PMS medium at the concentrations of $0.5 \mathrm{mM}$ or $5 \mathrm{mM}$, and 0.5 or 


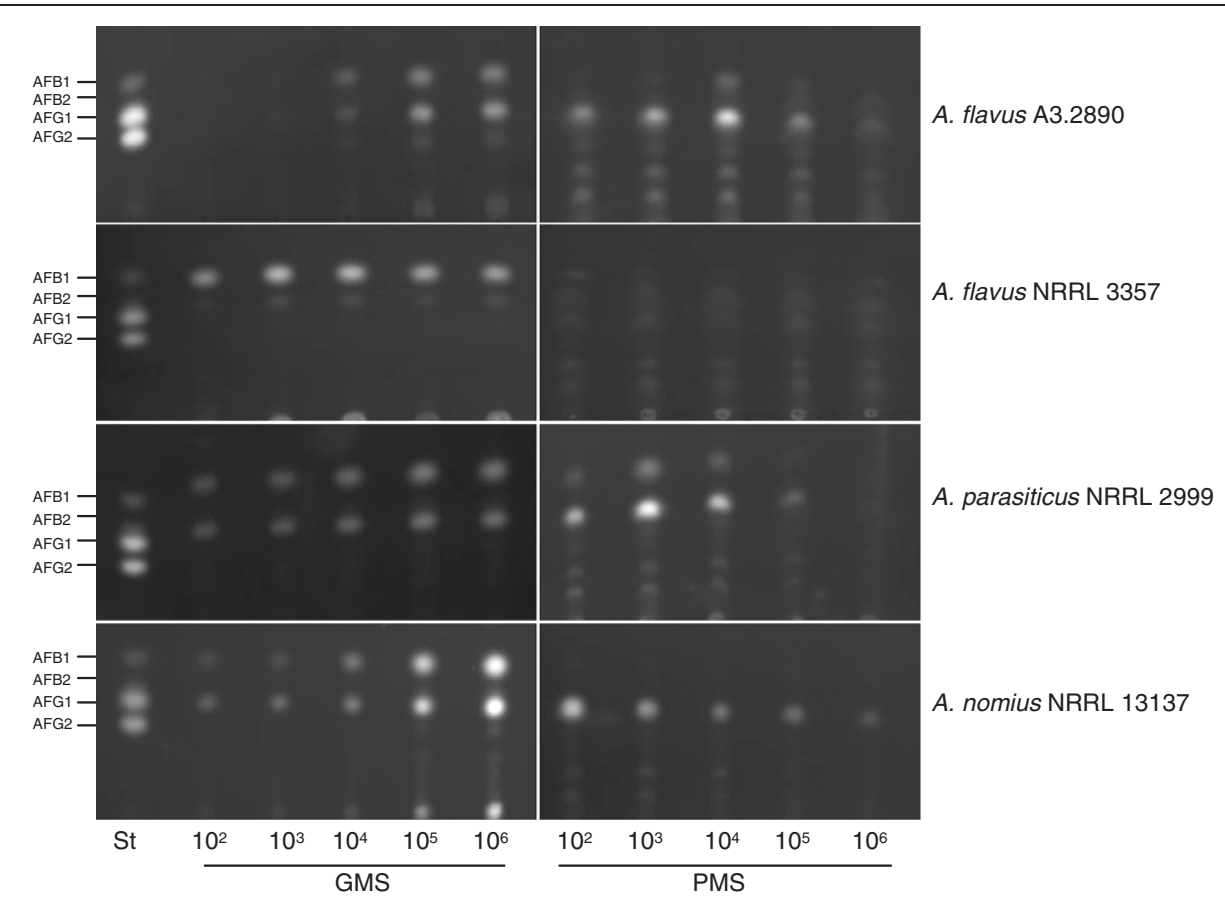

Figure 5 The density effect is present in all Aspergillus strains tested except $A$. flavus NRRL 3357. Strains of $A$. flavus NRRL 3357 , A parasiticus NRRL 2999 and A. nomius NRRL 13137 were tested for their density effects. Freshly prepared spores at the densities of $10^{2}$ to $10^{6}$ were cultured in GMS and PMS media and AF contents were analyzed by TLC after 3 days. Note no AF was produced in PMS media by A. flavus NRRL 3357. St: AF standards.

$5 \mathrm{mM} \mathrm{NaCl}$ was added to the culture as a control, and then performed liquid incubation with the final spore densities of $10^{4}$ or $10^{6}$ per ml using freshly prepared $A$. flavus A3.2890 spore suspensions. TLC analyses were performed for AFs extracted from the media. We observed that none of these treatments had any significant effect on AF production. No AF production was observed in any of the high initial spore density cultures (Figure 7). These results suggest that the inhibited AF biosynthesis in high initial spore density cultures was unlikely caused by reduced levels of TCA cycle intermediates.

\section{Discussion}

As a group of highly toxic natural compounds, AFs in nature are produced mainly in seeds with high lipid and protein content $[1,3]$. Previous reports show that peptone is not a suitable carbon source for AF production [23-25]. Our present study demonstrates that peptone was in fact conducive for AF production, as long as the initial spore density of $A$. flavus was reduced. Mycelia grown in peptone media responded not only to the initial spore density, but also to peptone concentration. Higher initial spore density and higher concentration of peptone inhibited AF biosynthesis. We also showed that no AF biosynthesis inhibitor was released into the media in the culture with the higher initial spore density. qRT-
PCR analyses revealed that culture with a high initial spore density repressed expression of both the transcriptional regulators and the biosynthesis genes in the AF pathway gene cluster. Metabolomic studies showed that, in high density cultures, the TCA cycle and PP pathway were active, while the fatty acid biosynthesis pathway was repressed.

\section{Spore density- and peptone concentration-dependent AF biosynthesis in PMS media}

In nature, many organisms, especially fungal species, are able to produce compounds to suppress the growth of other organisms in their neighborhood [51]. Regulated production of these compounds is expected to have physiological and ecological advantage for these organisms. It has been shown previously that lower glucose content supports fungal growth but not AF accumulation, suggesting that the first priority of the fungus is growth when food availability is low [27]. In our study we observed that mycelia grown in peptone media showed spore density- and peptone concentrationdependent AF production in A. flavus. High initial spore density or high peptone concentration promoted rapid mycelial growth without AF biosynthesis, which may allow the fungus to prioritize propagation when the competition pressure is low, and when sufficient food is available. In contrast, active AF productions were 

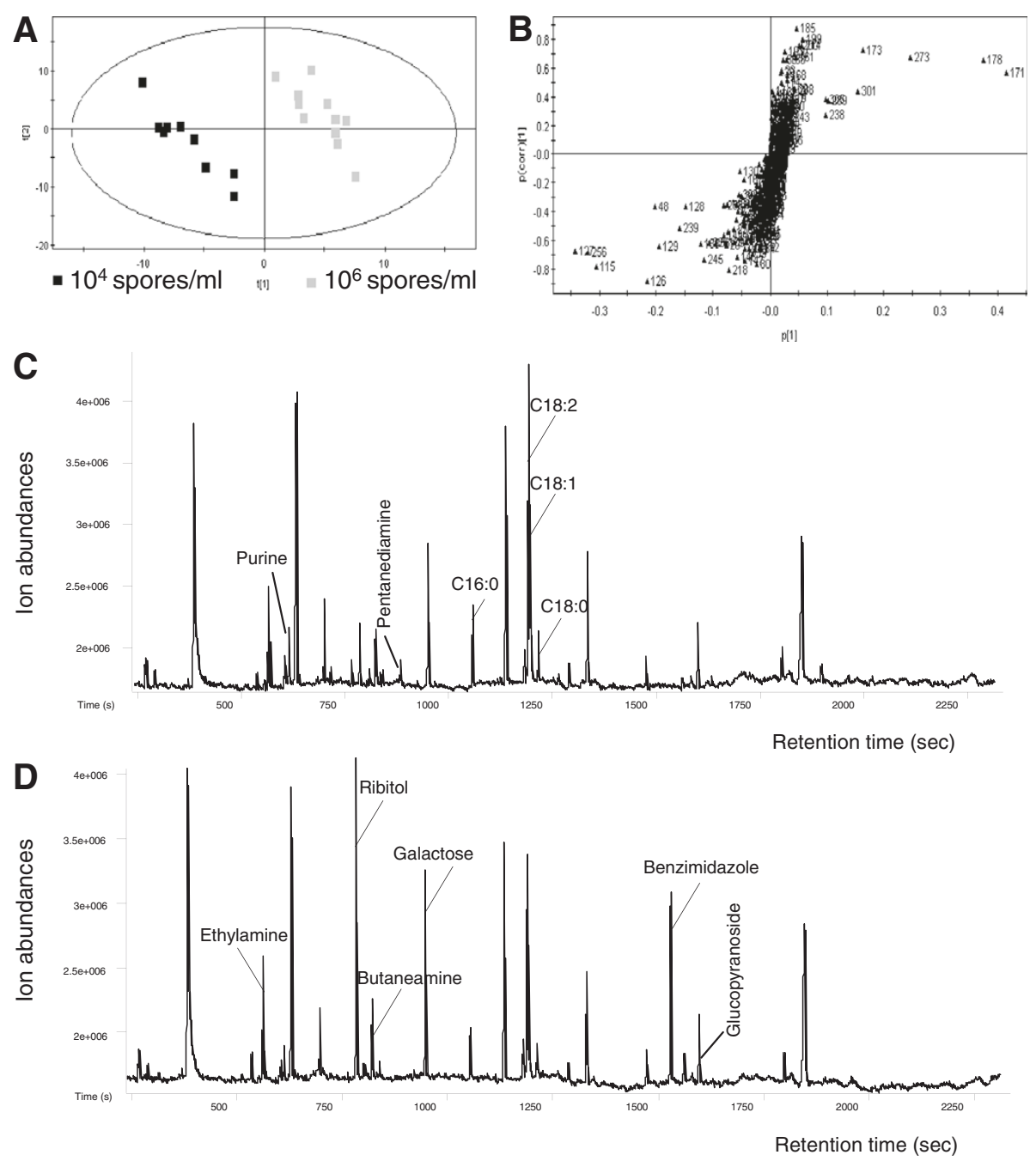

Figure 6 Metabolites with different contents in cultures initiated with high or low spore densities. (A) A PLS scores plot, performed using SIMCA-P V11.0, for metabolites extracted from mycelia cultured for 2, 3, 4 and 5 days in PMS media with initial spore densities of $10^{4}$ (black) and $10^{6}$ (gray) spores $/ \mathrm{ml}$, with 3 replicates in each treatment. (B) Scatter loading plots obtained from PLS analyses of the entire GC-Tof-MS dataset. (C and $\mathbf{D}$ ) Total ion chromatographies of metabolites extracted from mycelia of A. flavus grown in PMS media for 3 days with the initial spore densities of $10^{4}$ (C) and $10^{6}$ spores $/ \mathrm{ml}(\mathrm{D})$. Metabolites with significant differences in quantity between (C) and (D) are labeled.

observed in cultures initiated with lower spore densities and lower concentrations of peptone. Additional comparative studies using several AF-producing strains including A. flavus, A. parasiticus and A. nomius from the USDA ARS culture collection showed that the densitydependent AF biosynthesis in PMS media was present in all strains tested except A. flavus NRRL 3357. This particular strain did not produce any AFs in PMS media, as reported previously [52]. Furthermore, a positive correlation between AF production and initial spore density in GMS media was also not observed for this strain, implicating a different regulation mechanism is evolved. In natural ecosystems where the self population density is low and food is scarce, AF production may confer competitive advantages, through inhibition of the growth of other organisms. It would be interesting to examine if other fungal species also employ this survival strategy.

We showed that no soluble AF biosynthesis inhibitor was released from the high spore density culture to media by using spent medium experiments, suggesting that A. flavus A3.2890 is somehow able to sense the population density and adjust their growth and AF production through cell-autonomous machinery. Unlike Candidia albicans and Dictyostelium, where density factors are diffusible to media [53-55], we hypothesize that A. flavus may use a cell surface component to perceive such cultural density and nutrient signals. The possible role of $\mathrm{G}$ protein-mediated signaling [56] in this process is worth exploring. Alternatively, it has been reported that oxidative stress is a prerequisite for AF production 
Table 1 Metabolites differ between high and low spore density cultures

\begin{tabular}{|c|c|c|c|c|c|}
\hline Groups & Metabolites & $\begin{array}{l}\text { R. T. } \\
\text { (sec) }\end{array}$ & $\begin{array}{l}\text { Relative contents } \\
\text { in } 10^{4} \text { spores } / \mathrm{ml}^{*}\end{array}$ & $\begin{array}{l}\text { Relative contents } \\
\text { in } 10^{6} \text { spores } / \mathrm{ml}^{*}\end{array}$ & $\begin{array}{l}10^{4} / 10^{6} \\
\text { ratio }\end{array}$ \\
\hline \multirow[t]{3}{*}{ TCA cycle intermediates } & Malic acid & 587.5 & $0.0187 \pm 0.0001$ & $0.0098 \pm 0.0027$ & 1.91 \\
\hline & Fumaric acid & 1349.0 & $0.0869 \pm 0.0090$ & $0.0509 \pm 0.0043$ & 1.71 \\
\hline & Succinic acid & 269.8 & $0.0103 \pm 0.0020$ & $0.0043 \pm 0.0016$ & 2.4 \\
\hline \multirow[t]{6}{*}{ Amino acids } & Glycine & 417.0 & $0.0066 \pm 0.0028$ & $0.0034 \pm 0.0003$ & 1.94 \\
\hline & Phenylalanine & 713.7 & $0.1649 \pm 0.0330$ & $0.0084 \pm 0.0007$ & 19.63 \\
\hline & Proline & 410.0 & $0.0552 \pm 0.0179$ & $0.0099 \pm 0.0009$ & 5.58 \\
\hline & Valine & 331.5 & $0.0191 \pm 0.00028$ & $0.0088 \pm 0.0006$ & 2.17 \\
\hline & Tyrosine & 964.1 & $0.0430 \pm 0.0090$ & $0.0242 \pm 0.0027$ & 1.78 \\
\hline & Isoleucine & 404.0 & $0.0059 \pm 0.0017$ & $0.0042 \pm 0.0003$ & 1.4 \\
\hline \multirow[t]{4}{*}{ Fatty acids } & Palmitic acid & 1054.5 & $0.3447 \pm 0.037$ & $0.2640 \pm 0.011$ & 1.31 \\
\hline & Stearic acid & 1212.4 & $0.2218 \pm 0.027$ & $0.1402 \pm 0.0133$ & 1.58 \\
\hline & Oleic acid & 1193.0 & $0.0833 \pm 0.0024$ & $0.0744 \pm 0.0033$ & 1.12 \\
\hline & Linoleic acid & 1188.8 & $0.8959 \pm 0.0671$ & $0.6315 \pm 0.0554$ & 1.42 \\
\hline \multirow[t]{2}{*}{ Nucleotides } & Pyrimidine & 449.0 & $0.0154 \pm 0.0044$ & $0.0039 \pm 0.0004$ & 3.95 \\
\hline & Purine & 630.8 & $2.1901 \pm 0.3141$ & $1.3194 \pm 0.0221$ & 1.66 \\
\hline \multirow[t]{2}{*}{ AA metabolism } & Pentanediamine & 881.1 & $0.1703 \pm 0.0143$ & $0.0162 \pm 0.0011$ & 10.51 \\
\hline & Amine & 369.0 & $0.0734 \pm 0.0261$ & $0.0328 \pm 0.0036$ & 2.24 \\
\hline \multirow[t]{7}{*}{ Sugar metabolism } & Ribitol & 784.2 & $0.6276 \pm 0.1768$ & $2.6039 \pm 0.1502$ & 0.24 \\
\hline & Glucopyranoside & 1561.8 & $0.1130 \pm 0.0198$ & $0.3344 \pm 0.0354$ & 0.34 \\
\hline & Gluconolactone-6-P & 821.6 & $0.5679 \pm 0.0839$ & $0.7094 \pm 0.0181$ & 0.80 \\
\hline & Glycerol & 986.0 & $0.0073 \pm 0.0015$ & $0.0103 \pm 0.0009$ & 0.71 \\
\hline & Butanediamine & 801.1 & $0.0656 \pm 0.0086$ & $0.1224 \pm 0.0051$ & 0.54 \\
\hline & Ehylamine & 563.0 & $0.2082 \pm 0.0320$ & $0.2436 \pm 0.0013$ & 0.85 \\
\hline & Galactose & 948.1 & $1.6122 \pm 0.4037$ & $1.9547 \pm 0.3306$ & 0.82 \\
\hline
\end{tabular}

*Values represent metabolites abundances (mean \pm SD) of 3 replicates from mixed 3 independent samples, as measured by GC-Tof-MS in 3-day mycelia (normalized by C17:0 fatty acid). R.T.: retention time; TCA: tricarboxylic acid; AA: amino acid.

[57]. It is plausible that the rapid growth in PMS media with high initial spore densities may lead to reduced intracellular oxygen availability and subsequently decreased oxidative stress, which could prevent AF production. It will be interesting to examine why this density-sensing machinery is active only when peptone, not glucose, is used as the carbon source.
High initial spore densities repressed expression of AF biosynthesis- related genes including afIS and afIR Transferring A. parasiticus mycelia from PMS to GMS media resulted in AF production, which is inhibited by cycloheximide or actinomycin D treatments, suggesting both de novo transcription and translation are required for AF biosynthesis $[23,24]$. In this study, we observed

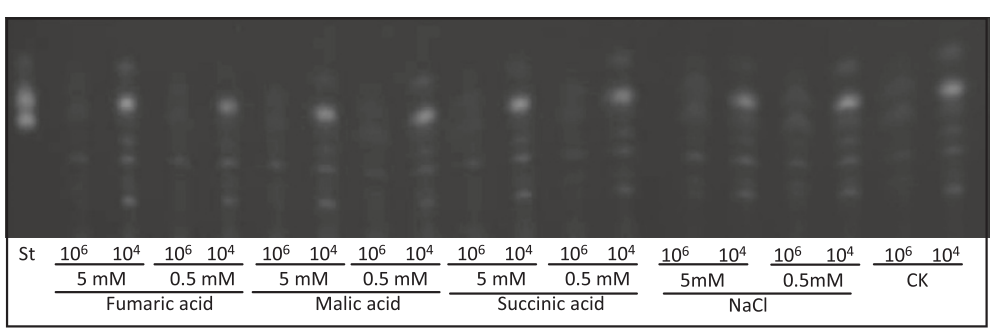

Figure 7 Additions of TCA cycle intermediates can not restore the AF biosynthesis in high initial spore density cultures. In A. flavus A3.2890 mycelia grown in PMS media initiated with $10^{4}$ and $10^{6}$ spores $/ \mathrm{ml}, 0.5 \mathrm{mM}$ or $5 \mathrm{mM}$ TCA cycle intermediates, fumaric acid, malic acid and succinic acid, were added at the beginning of the culture. AFs were extracted from media and analyzed by TLC after 3-day cultivations. 
that high initial spore densities promoted mycelial growth but inhibited AF production, which is similar to the high temperature cultures in GMS media where no AFs are produced [58]. High temperature culture $\left(37^{\circ} \mathrm{C}\right)$ specifically represses the expressions of AF biosynthesis genes without affecting expression of the transcriptional regulators aflR and aflS in the AF pathway gene cluster $[20,59,60]$. However, we found that high initial density cultures inhibited the expression of both the transcriptional regulators (aflR and aflS) and downstream AF biosynthesis genes simultaneously, suggesting a different manner of regulation. Further study is needed to elucidate if the density-dependent AF biosynthesis is regulated through antagonistic signaling pathways that coordinate vegetative growth, conidiation and AF production [2].

\section{Cultures with high initial spore densities in PMS media trigger a metabolic shift from AF production to sugar metabolism}

Although primary and secondary metabolism share common transcriptional and translational machinery, secondary metabolism often commences during idiophase, when normal growth and development have ceased [61]. The present study revealed a novel low cell density-dependent metabolic switch toward AF production in A. flavus. We observed that the AF and lipid biosynthesis were active in mycelia initiated with a low spore density in the PMS medium. In contrast, the TCA cycle was inhibited, as shown by the accumulation of TCA cycle intermediates in low spore density cultures, which is in agreement with previous results showing that the TCA cycle is repressed during active AF biosynthesis, to allow a greater acetyl-CoA shunt toward AF biosynthesis [26]. By adding three TCA cycle intermediates to cultures, we showed that increased TCA cycle intermediates did not restore AF biosynthesis in the high initial spore density culture, nor did additional TCA cycle intermediates promote AF biosynthesis in the low spore density culture, suggesting that the spore densityregulated AF biosynthesis in the PMS medium is not likely influenced by TCA cycling directly. The enhanced TCA cycling might be the consequences of inhibited AF biosynthesis in the high spore density culture.

Since AF production shares a subset of biosynthetic steps with fatty acid metabolism, accumulation of AFs and lipids often occur in parallel $[18,62]$. This parallel biosynthesis trend was observed in our Metabolomic studies. All four fatty acids detected, palmitic acid, stearic acid, oleic acid and linoleic acid, were accumulated in the low spore density culture, together with AF biosynthesis. The density-dependent metabolic switch from active TCA cycling in high initial spore density cultures to active AF biosynthesis in low initial spore density cultures may represent a shift in metabolic priority that allows A. flavus to produce AFs in the protein-rich environment only when its own population density is low.

\section{Conclusions}

Our studies demonstrate that A. flavus grown in media with peptone as the carbon source is able to detect its own population density and nutrient availability, and is able to switch between fast growth and AF production. High initial spore density or high peptone concentration led to rapid mycelial growth and inhibited AF production, while low initial spore density or low peptone concentration promoted AF biosynthesis. Inhibited $\mathrm{AF}$ biosynthesis in the high initial spore density culture was accompanied by active TCA cycling and rapid mycelial growth. Supplements of TCA cycle intermediates did not restore AF biosynthesis, suggesting the inhibited AF biosynthesis was not caused by depletion of TCA intermediates. Our spent medium experiments showed that the density-sensing factor regulates AF biosynthesis in a cell-autonomous manner. Expression analyses showed that the density factor acts at the transcriptional level to regulate the expressions of both aflR and aflS transcription regulators and downstream AF biosynthesis genes. Interestingly, Most Aspergillus strains including A parasiticus and $A$. nomius tested were shown to be densitydependent AF biosynthesis in PMS media. Only $A$. flavus NRRL 3357 did not exhibit the density-dependent AF biosynthesis, suggesting different regulation machinery is evolved in this strain. We believe that a cell density- or peptone availability-dependent metabolic switch may provide $A$. flavus with a competitive advantage in the natural ecosystem. Whether or not the perception of population density and peptone availability are regulated through the same signaling pathway will require further study.

\section{Methods}

\section{Fungal strain and growth conditions}

The primary strain used in this study, A. flavus A3.2890, was obtained from CGMCC, located in the Institute of Microbiology, Chinese Academy of Sciences. A. flavus NRRL 3357, A. parasiticus NRRL 2999 and A. nomius NRRL 13137 strains were obtained from the ARS culture collection in USDA. The GMS medium was prepared as previously described [63], which contains $50 \mathrm{~g} / \mathrm{L}$ glucose, $3 \mathrm{~g} / \mathrm{L}(\mathrm{NH} 4)_{2} \mathrm{SO} 4,2 \mathrm{~g} / \mathrm{L} \mathrm{MgSO} 4,10 \mathrm{~g} / \mathrm{L} \mathrm{KH}_{2} \mathrm{PO} 4$, and $1 \mathrm{ml} / \mathrm{L}$ trace element mixture. The $\mathrm{pH}$ was adjusted to 4.5 before autoclaving. The PMS medium was identical to GMS except the glucose was replaced by $5 \%$ peptone, and $\mathrm{pH}$ was adjusted to 5.2, as described previously [24]. All cultures were prepared by following Park's protocol [64] with minor modifications. Sixty $\mu \mathrm{l}$ of $A$. flavus spore suspensions stored at $-80^{\circ} \mathrm{C}$ in glycerol was pre-cultured 
on potato-dextrose agar plates at $37^{\circ} \mathrm{C}$ for 4 days. Mature spores on the surface were harvested and resuspended in sterile distilled water containing $0.05 \%$ Tween 20 (Sigma, St. Louis, USA), diluted to a series of spore densities after counting with a haemacytometer. Five $\mathrm{ml}$ of spore suspensions of desired density were added to $45 \mathrm{ml}$ PMS or GMS liquid media, cultured on a shaker $(180 \mathrm{rpm})$ at $28^{\circ} \mathrm{C}$ in the dark.. The $\mathrm{pH}$ of the culture media was measured at different time points following inoculation, during a 55-hr culture period. The three brands of peptone used in this study were purchased from Sigma (Cat. No. P6463, St. Louis, USA), Beijing Aoboxing Biotech (Cat. No. 01-001, Beijing, China) and Beijing Shuangxuan Microbe Culture Medium Products Factory (Cat. No. 02-31A, Beijing, China). TCA cycle intermediates, fumaric acid (Cat. No. F8509), malic acid (Cat. No. M1210) and succinic acid (Cat. No. S3674), were purchased from Sigma-Aldrich and added to PMS media at the beginning of the culture.

\section{Determinations of fungal dry weights and AF contents}

For the determination of fungal dry weights, mycelia grown in $50 \mathrm{ml}$ media were harvested at different time points $(48,72,96,120 \mathrm{hrs}$ after inoculations) by filtration through two layers of filter paper, washed by sterilized water, and then freezer-dried before weighing. The filtrate was sterilized by passing through a $0.22 \mu \mathrm{m}$ membrane, which was used for spent media experiments and AF quantifications. For extraction of AFs from media, an equal volume of chloroform was added and the mixture was vortexed and extracted ultrasonically for $15 \mathrm{~min}$. After centrifugation for 6 minutes at $11498.6 x g$, the organic phase (lower phase) was filtered through a $0.22 \mu \mathrm{m}$ membrane and dried under nitrogen gas flow, and re-dissolved in fixed volumes of chloroform. The extracts were analyzed by TLC as previously described [65], except the developing solvent was changed to $\mathrm{CHCl}_{3}: \mathrm{H}_{2} \mathrm{O}(9: 1, \mathrm{v} / \mathrm{v})$. The AF levels were quantified by HPLC (Agilent 1200, Waldbronn, Germany), equipped with a reverse phase C18 column $(150 \mathrm{~mm}$ in length and $4.6 \mathrm{~mm}$ internal diameter, $5 \mu \mathrm{m}$ particle size; Agilent), eluted by gradient elution, starting with a mixture of $25 \%$ methanol, $20 \%$ acetonitrile and 55\% water for $3 \mathrm{~min}$, then changed to a $38 \%$ methanol water solution for $0.1 \mathrm{~min}$, eluted with $38 \%$ methanol for $2.9 \mathrm{~min}$, detected by a DAD analyzer at $360 \mathrm{~nm}$. Quantification was performed by calculating the amount of AF in samples from a standard calibration curve. For the detection of AFs from the mycelia, dried mycelia were ground to a powder, then extracted with acetone with solid-to-liquid ratio $1: 10(\mathrm{~g} / \mathrm{ml})$ for 30 minutes, the extract was analyzed by TLC as described above.

\section{Metabolomic analyses by GC-Tof-MS}

Mycelia harvested from the $2^{\text {nd }}$ to the $5^{\text {th }}$ day with a 24 $\mathrm{hr}$ interval were lyophilized and extracted by ultrasonication for $40 \mathrm{~min}$ with $1.5 \mathrm{ml}$ mixed solvents including methanol, chloroform and water $(5: 2: 1, \mathrm{v} / \mathrm{v} / \mathrm{v})$, in which $100 \mu \mathrm{l}$ of $1 \mathrm{mg} / \mathrm{ml}$ heptadecanoic acid (C17:0, Sigma, St. Louis, USA) was added as an internal standard. After the centrifugation at 11,000 $\mathrm{g}$ for $10 \mathrm{~min}, 1 \mathrm{ml}$ of supernatant was transferred to a tube with $400 \mu \mathrm{l}$ chloroform and $400 \mu \mathrm{l}$ water, vortexed for $15 \mathrm{sec}$, centrifuged at 11498.6xg for $10 \mathrm{~min}$, and then $400 \mu \mathrm{l}$ chloroform phase was transferred to a new glass vial, and dried under the nitrogen gas flow. The pellet was re-dissolved in $50 \mu \mathrm{l}$ $20 \mathrm{mg} / \mathrm{ml} \mathrm{O}$-methylhydroxylamin hydrochloride (Sigma, Steinheim, Switzerland) in pyridine, vortexed and incubated at $37^{\circ} \mathrm{C}$ for $120 \mathrm{~min}$. Afterwards, $100 \mu \mathrm{l} \mathrm{N}$-methylN-trimethylsily trifluoroacetamide (Sigma, Steinheim, Switzerland) was added immediately to the mixture, vortexed and incubated at $37^{\circ} \mathrm{C}$ on a shaker $(150 \mathrm{rpm})$ for $30 \mathrm{~min}$, The silyl-derivatized samples were analyzed by GC-Tof-MS after cooling to the room temperature using an Agilent 6890 gas chromatography coupled to a LECO Pegasus IV GC-Tof-MS (LECO, USA) with the EI ionization. The column used was VF-5 ms $(30 \mathrm{~m}$ in length; $250 \mu \mathrm{m}$ internal diameter, $0.25 \mu \mathrm{m}$ film thickness; Varian, USA). The MS was operated in a scan mode (start after $4 \mathrm{~min}$; mass range: $50-700 \mathrm{~m} / \mathrm{z}$; $2.88 \mathrm{sec} / \mathrm{scan}$; detector voltage: $1400 \mathrm{~V}$ ), in which helium was used as the carrier gas $(1 \mathrm{ml} / \mathrm{min})$ with a constant flow mode, a split injector $\left(340^{\circ} \mathrm{C}, 1: 50\right.$ split $)$ and a flame ionization detector $\left(340^{\circ} \mathrm{C}\right)$. The samples were subjected to a column temperature of $100^{\circ} \mathrm{C}$ for $3 \mathrm{~min}$, raised to $150^{\circ} \mathrm{C}$ at a rate of $10^{\circ} \mathrm{C} / \mathrm{min}$, then to $250^{\circ} \mathrm{C}$ at $5^{\circ} \mathrm{C} / \mathrm{min}$, finally to $360^{\circ} \mathrm{C}$ at $10^{\circ} \mathrm{C} / \mathrm{min}$, and held for $15 \mathrm{~min}$ at $360^{\circ}$ C. Sample components were identified by comparison of retention times and mass spectra with reference compounds, and matching to the NIST mass spectral database. Metabolite peak areas representing the abundance of metabolites were normalized to the internal standard (heptadecanoic acid). Multivariate analysis was performed using SIMCA-P V11.5 (Umetrics, Sweden) $[66,67]$. All GC-Tof-MS analyses were conducted with three replicate cultures, mixed before extractions, and measured three times to get the average contents.

\section{Expression analyses using qRT-PCR}

Mycelia were harvested, frozen and ground in liquid nitrogen. Total RNAs from the mycelia were extracted using Trizol (Invitrogen, USA), and polyA mRNAs were purified using PolyAT Rack mRNA Isolation System (Promega, Madison, WI) according to the manufacturers' manual. All cDNAs were synthesized by reverse transcription reaction performed with ReverTra Ace (Toyobo, Japan) at $42^{\circ} \mathrm{C}$ for $1 \mathrm{~h}$, and then $85^{\circ} \mathrm{C}$ for 
15 min to stop the reaction. qRT-PCRs were performed using SYBR Green I in a Rotor-Gene 3000 Cycler (Corbett Research, Australia) with primers and temperatures as described in Additional file 7.

\section{Additional files}

\section{Additional file 1: Alignment of ITS sequence of the $A$. flavus} A3.2890 with ITS sequences from 13 different Aspergillus species in GenBank. The Genbank accession numbers for ITS sequences used are $A$. flavus: AF138287.1, A. parasiticus: GU953212.1, A. sojae: AB008419.1, A tamari: JF901808.1 A. pseudotamarii: DQ467986.1, A. caelatus: EU645658.1, A. nomius: AF027860, A. bombycis: AF338740, A. niger: JN545800, A. arachidicola: HM560045, A. fumigatus: JN153038, A. terreus: EF568102, and A. nidulans: AF138289.1

Additional file 2: Homology matrix and phylogenetic tree, calculated based on comparison among the ITS sequence of $A$. flavus A3.2890 and sequences from different Aspergillus species in GenBank. Note that within the 529 bp region, the ITS sequence of $A$. flavus A3.2890 showed $99.6 \%$ identity with the corresponding sequence from $A$. flavus (with only 2 SNPs in the entire region), followed by those from A. parasiticus (98.7\%), A. sojae (98.5\%), A. tamari (98.1\%), and A. pseudotamarii and $A$. caelatus (97.9\%). Note that $97.7 \%$ sequence identity was observed between the ITS sequence from A. flavus A3.2890 and that from $A$. arachidicola that also produces both AFB and AFG (with 15 SNPS in the same region).

Additional file 3: Alignment and homology matrix of the calmodulin sequence of the $A$. flavus A3.2890 with calmodulin sequences from 19 different Aspergillus species in GenBank. Note that the calmodulin sequence from A. flavus A3.2890 showed the highest homology with the calmodulin genes from $A$. flavus and A. kambarensis, while A. kambarensis is known to be synonymous to $A$. flavus, but without AF production (Varga et al., 2011).

Additional file 4: Alignment and homology matrix of the betatubulin sequence of the $A$. flavus A3.2890 with beta-tubulin sequences from 14 different Aspergillus species in GenBank. The beta-tubulin sequence from A. flavus A3.2890 showed the highest homology with the beta-tubulin genes from $A$. flavus, A. fasciculatus, A. oryzae, A. subolivaceus and A. kambarensis. Note that beta-tubulin genes are less effective in discriminating these closely related strains, as observed by Varga et al. (2011)

Additional file 5: Evaluation of peptone from different suppliers. AF productions, as showed by TLC analyses, by A. flavus A3.2890 cultured in PMS (B) media made by peptone from 3 different sources for 3 days with the initial spore densities of $10^{2}, 10^{4}$, and $10^{6}$ spores $/ \mathrm{ml}$. Three brands of peptone were purchased from Aoboxing, Sigma and Shuangxuan.

Additional file 6: AF contents in mycelia of A. flavus A3.2890 cultured in PMS and GMS media. In PMS media, high initial spore density led to reduced AF contents in mycelia, while in GMS media high initial spore density led to increased AF contents in mycelia. The AFs were extracted from mycelia after 3-day incubation. P4 and P6: mycelia cultured in PMS media with initial spore densities of $10^{4}$ and $10^{6}$ spore/ $\mathrm{ml}$, respectively; G4 and G6: mycelia cultured in GMS media with initial spore densities of $10^{4}$ and $10^{6}$ spores $/ \mathrm{ml}$, respectively.

Additional file 7: Primers and PCR schemes used for qRT-PCR analyses.

\section{Competing interest}

The authors declare that they have no competing interests.

\section{Acknowledgments}

We thank Fen Yang and Fang Chen for early protocol development, Lixin Duan and Zhen Xue at the Key Laboratory of Plant Molecular Physiology for technical assistance, John Snyder for critical reading of the manuscript, and Fuzeng Hong and Zhizhong Cao at Practaculture College of Gansu Agricultural University for suggestions. This work is supported by the CAS/
SAFEA International Partnership Program for Creative Research Teams (20090491019), Key Innovation Project (KSCX2-YW-N-033) and the NNSF Innovative Research Team Project (31121065).

\section{Author details}

${ }^{1}$ Practaculture College, Gansu Agricultural University, Lanzhou 730070, China. ${ }^{2}$ Key Laboratory of Plant Molecular Physiology, Institute of Botany, Chinese Academy of Sciences, 20 Nanxincun, Fragrant Hill, Beijing, 100093, China.

${ }^{3}$ School of Biology and Basic Medical Sciences, Soochow University, Suzhou 215000, China.

\section{Authors' contributions}

SY performed most of the experiments, and drafted the manuscript. YL carried out the comparative studies for different strains and experiments for TCA cycle intermediates treatments. JZ carried out the qRT-PCR and molecular characterization of the A3.2890 strain used in this study. CML supervised the study, participated in experimental design, and revised the manuscript. All authors read and approved the final manuscript.

Received: 2 December 2011 Accepted: 13 June 2012

Published: 13 June 2012

\section{References}

1. Reddy MJ, Shetty HS, Fanelli C, Lacey J: Role of seed lipids in Aspergillus parasiticus growth and aflatoxin production. J Sci Food Agric 1992, 59(2):177-181

2. $\mathrm{Yu} \mathrm{JH}$, Keller NP: Regulation of secondary metabolism in filamentous fungi. Annu Rev Phytopathol 2005, 43:437-458.

3. Molyneux RJ, Mahoney N, Kim JH, Campbell BC: Mycotoxins in edible tree nuts. Int J Food Microbiol 2007, 119(1-2):72-78.

4. Bennett JW, Klich M: Mycotoxins. Clin Microbiol Rev 2003, 16(3):497-516

5. Bhatnagar D, Ehrlich K, Cleveland T: Molecular genetic analysis and regulation of aflatoxin biosynthesis. Appl Microbiol Biotech 2003, 61(2):83-93.

6. Georgianna DR, Payne GA: Genetic regulation of aflatoxin biosynthesis: from gene to genome. Fungal Genet Biol 2009, 46(2):113-125.

7. Liu BH, Chu FS: Regulation of afIR and its product, AfIR, associated with aflatoxin biosynthesis. Appl Environ Microbiol 1998, 64(10):3718-3723.

8. Yu J, Chang PK, Ehrlich KC, Cary JW, Bhatnagar D, Cleveland TE, Payne GA, Linz JE, Woloshuk CP, Bennett JW: Clustered pathway genes in aflatoxin biosynthesis. Appl Environ Microbiol 2004, 70(3):1253-1262.

9. Bayram Ö, Krappmann S, Ni M, Bok JW, Helmstaedt K, Valerius O, BrausStromeyer S, Kwon NJ, Keller NP, Yu JH: VelB/VeA/LaeA complex coordinates light signal with fungal development and secondary metabolism. Science 2008, 320(5882):1504-1506.

10. Calvo AM: The VeA regulatory system and its role in morphological and chemical development in fungi. Fungal Genet Biol 2008, 45(7):1053-1061.

11. Buchanan RL, Stahl HG: Ability of various carbon sources to induce and support aflatoxin synthesis by Aspergillus parasiticus. J Food Saf 1984, 6(4):271-279.

12. Kachholz T, Demain AL: Nitrate repression of averufin and aflatoxin biosynthesis. J Nat Prod 1983, 46(4):499-506.

13. Aziz NH, Moussa LA: Influence of white light, near-UV irradiation and other environmental conditions on production of aflatoxin B1 by Aspergillus flavus and ochratoxin A by Aspergillus ochraceus. Mol Nutr Food Res 1997, 41(3):150-154

14. Joffe $A Z$, Lisker $\mathrm{N}$ : Effects of light, temperature, and $\mathrm{pH}$ value on aflatoxin production in vitro. App/ Microbio/ 1969, 18(3):517-518.

15. Trenk HL, Hartman PA: Effects of moisture content and temperature on aflatoxin production in corn. Appl Microbio/ 1970, 19(5):781-784.

16. Buchanan $\mathrm{RL}$, Ayres JC: Effect of initial $\mathrm{pH}$ on aflatoxin production. App/ Microbiol 1975, 30(6):1050-1051.

17. Clevstrom G, Ljunggren $\mathrm{H}$, Tegelstrom S, Tideman K: Production of aflatoxin by an Aspergillus flavus isolate cultured under a limited oxygen supply. Appl Environ Microbiol 1983, 46(2):400-405.

18. Shih CN, Marth EH: Aflatoxin formation, lipid synthesis, and glucose metabolism by Aspergillus parasiticus during incubation with and without agitation. Biochim Biophys Acta 1974, 338(1):286-296.

19. Watanabe $\mathrm{CMH}$, Townsend $\mathrm{CA}$ : Incorporation of molecular oxygen in aflatoxin B1 biosynthesis. J Org Chem 1996, 61(6):1990-1993. 
20. Price MS, Shannon BCB, Sabrina TB, Robert AKB, Payne GA: Aflatoxin conducive and non-conducive growth conditions reveal new gene associations with aflatoxin production. Fungal Genet Biol 2005, 42(6):506-518

21. Wilkinson J, Yu J, Abbas H, Scheffler B, Kim H, Nierman W, Bhatnagar D, Cleveland T: Aflatoxin formation and gene expression in response to carbon source media shift in Aspergillus parasiticus. Food Addit Contam 2007, 24(10):1051-1060.

22. Davis ND, Diener UL: Growth and aflatoxin production by Aspergillus parasiticus from various carbon sources. Appl Environ Microbiol 1968, 16(1):158-159

23. Abdollahi A, Buchanan RL: Regulation of aflatoxin biosynthesis: characterization of glucose as apparent inducer of aflatoxin production. J Food Sci 1981, 46(2):633-635.

24. Abdollahi A, Buchanan RL: Regulation of aflatoxin biosynthesis: induction of aflatoxin production by various carbohydrates. J Food Sci 1981, 46(1):143-146.

25. Buchanan RL, Lewis DF: Regulation of aflatoxin biosynthesis: effect of glucose on activities of various glycolytic enzymes. Appl Environ Microbiol 1984, 48(2):306-310.

26. Shantha T, Murthy VS: Influence of tricarboxylic acid cycle intermediates and related metabolites on the biosynthesis of aflatoxin by resting cells of Aspergillus flavus. App/ Environ Microbiol 1981, 42(5):758-761.

27. Wiseman DW, Buchanan RL: Determination of glucose level needed to induce aflatoxin production in Aspergillus parasiticus. Can J Microbiol 1987, 33(9):828-830.

28. Amaike S, Keller NP: Distinct roles for VeA and LaeA in development and pathogenesis of Aspergillus flavus. Eukaryot Cell 2009, 8(7):1051-1060.

29. Brown SH, Scott JB, Bhaheetharan J, Sharpee WC, Milde L, Wilson RA, Keller NP: Oxygenase coordination is required for morphological transition and the host-fungus interaction of Aspergillus flavus. Mol Plant-Microbe Interact 2009, 22(7):882-894.

30. Brown RL, Cotty P, Cleveland TE, Widstrom N: Living maize embryo influences accumulation of aflatoxin in maize kernels. J Food Prot 1993, 56(11):967-971.

31. Keller NP, Butchko R, Sarr B, Phillips TD: A visual pattern of mycotoxin production in maize kernels by Aspergillus spp. Phytopathology 1994, 84(5):483-488

32. Jay E, Cotty PJ, Dowd MK: Influence of lipids with and without other cottonseed reserve materials on aflatoxin $\mathrm{B} 1$ production by Aspergillus flavus. J Agric Food Chem 2000, 48(8):3611-3615.

33. Calvo AM, Hinze LL, Gardner HW, Keller NP: Sporogenic effect of polyunsaturated fatty acids on development of Aspergillus spp. Appl Environ Microbiol 1999, 65(8):3668-3673.

34. Burow GB, Gardner HW, Keller NP: A peanut seed lipoxygenase responsive to Aspergillus colonization. Plant Mol Biol 2000, 42(5):689-701.

35. Maggio-Hall LA, Wilson RA, Keller NP: Fundamental contribution of $\beta$-oxidation to polyketide mycotoxin production in planta. Mol Plant-Microbe Interact 2005, 18(8):783-793.

36. Tsitsigiannis DI, Kunze S, Willis DK, Feussner I, Keller NP: Aspergillus infection inhibits the expression of peanut 13S-HPODE-forming seed lipoxygenases. Mol Plant-Microbe Interact 2005, 18(10):1081-1089.

37. Hu LB, Shi ZQ, Zhang T, Yang ZM: Fengycin antibiotics isolated from B-FS01 culture inhibit the growth of Fusarium moniliforme Sheldon ATCC 38932. FEMS Microbiol Lett 2007, 272(1):91-98.

38. Zhang $B$, Wang DF, Wu H, Zhang $L, X u Y$ : Inhibition of endogenous a-amylase and protease of Aspergillus flavus by trypsin inhibitor from cultivated and wild-type soybean. Ann Microbiol 2010, 60(3):405-414.

39. Zhang $T$, Shi ZQ, Hu LB, Cheng LG, Wang F: Antifungal compounds from Bacillus subtilis B-FS06 inhibiting the growth of Aspergillus flavus. World J Microbiol Biotechnol 2008, 24(6):783-788.

40. Vaamonde G, Patriarca A: Fernandez Pinto V, Comerio R, Degrossi C: Variability of aflatoxin and cyclopiazonic acid production by Aspergillus section Flavi from different substrates in Argentina. Intl J Food Microbiol 2003, 88(1):79-84.

41. Varga J, Frisvad JC, Samson RA: A reappraisal of fungi producing aflatoxins. World Mycotoxin J 2009, 2(3):263-277.

42. Varga J, Frisvad J, Kocsube S, Brankovics B, Toth B, Szigeti G, Samson R: New and revisited species in Aspergillus section Nigri. Stud Mycol 2011, 69(1):1-17.
43. Henry T, Iwen PC, Hinrichs SH: Identification of Aspergillus species using internal transcribed spacer regions 1 and 2. J Clin Microbiol 2000, 38(4):1510-1515.

44. Rodrigues P, Santos C, Venâncio A, Lima N: Species identification of Aspergillus section Flavi isolates from Portuguese almonds using phenotypic, including MALDI-TOF ICMS, and molecular approaches. $J$ Appl Microbiol 2011, 111:877-892.

45. Odds F, Hall C, Abbott A: Peptones and mycological reproducibility. Med Mycol 1978, 16(4):237-246.

46. Buchanan RL, Jones SB, Stahl HG: Effect of miconazole on growth and aflatoxin production by Aspergillus parasiticus. Mycopathologia 1987, 100(3):135-144.

47. Cai JJ, Zeng HM, Shima Y, Hatabayashi H, Nakagawa H, Ito Y, Adachi $Y$, Nakajima H, Yabe K: Involvement of the nadA gene in formation of G-group aflatoxins in Aspergillus parasiticus. Fungal Genet Biol 2008, 45(7):1081-1093

48. Wicklow DT, Shotwell OL, Adams GL: Use of aflatoxin-producing ability medium to distinguish aflatoxin-producing strains of Aspergillus flavus. Appl. Environ. Microbiol 1981, 41(3):697-699.

49. Tan KC, Trengove RD, Maker GL, Oliver RP, Solomon PS: Metabolite profiling identifies the mycotoxin alternariol in the pathogen Stagonospora nodorum. Metabolomics 2009, 5(3):330-335.

50. Ipcho SVS, Tan KC, Koh G, Gummer J, Oliver RP, Trengove RD, Solomon PS: The transcription factor StuA regulates central carbon metabolism, mycotoxin production, and effector gene expression in the wheat pathogen Stagonospora nodorum. Eukaryot Cell 2010, 9 (7):1100-1108.

51. Reverberi M, Ricelli A, Zjalic S, Fabbri AA, Fanelli C: Natural functions of mycotoxins and control of their biosynthesis in fungi. Appl Microbiol Biotechnol 2010, 87(3):899-911.

52. Woloshuck CP, Foutz KR, Brewer JF, Bhatnagar D, Cleveland TE, Payne GA: Molecular characterization of aflR, a regulatory locus for aflatoxin biosynthesis. Appl. Environ. Microbio/ 1994, 60(7):2408-2414.

53. Clarke M, Kayman SC, Riley K: Density-dependent induction of discoidinsynthesis in exponentially growing cells of Dictyostelium discoideum. Differentiation 1987, 34(2):79-87.

54. Jain R, Yuen I, Taphouse C, Gomer R: A density-sensing factor controls development in Dictyostelium. Genes Dev 1992, 6(3):390-400.

55. Lo HJ, Kohler JR, DiDomenico B, Loebenberg D, Cacciapuoti A, Fink GR: Nonfilamentous C. albicans mutants are avirulent. Cell 1997, 90(5):939-949.

56. Versele M, De Winde JH, Thevelein JM: A novel regulator of $G$ protein signaling in yeast, Rgs2, downregulates glucose-activation of the CAMP pathway through direct inhibition of Gpa2. EMBO J 1999, 18(20):5577-5591.

57. Jayashree T, Subramanyam C: Oxidative stress as a prerequisite for aflatoxin production by Aspergillus parasiticus. Free Radic Biol Med 2000, 29(10):981-985.

58. Schroede HW, Palmer JG, Eisenberg W: Aflatoxin production by Aspergillus flavus as related to various temperatures. Appl Microbiol 1967, 15(5):1006.

59. Obrian GR, Georgianna DR, Wilkinson JR, Yu J, Abbas HK, Bhatnagar D, Cleveland TE, Nierman W, Payne GA: The effect of elevated temperature on gene transcription and aflatoxin biosynthesis. Mycologia 2007, 99(2):232-239.

60. Schmidt-Heydt M, Magan N, Geisen R: Stress induction of mycotoxin biosynthesis genes by abiotic factors. FEMS Microbiol Lett 2008, 284(2):142-149.

61. Behal V: Enzymes of secondary metabolism in microorganisms. Trends Biochem Sci 1986, 11(2):88-91.

62. Hopwood DA: Molecular genetics of polyketides and its comparison to fatty acid biosynthesis. Annu Rev Genet 1990, 24:37-66.

63. Adye J, Mateles R: Incorporation of labelled compounds into aflatoxins. Biochim Biophys Acta 1964, 86:418-420.

64. Park JC, Nemoto Y, Homma T, Sato R, Matsuoka H, Ohno H, Takatori K, Kurata $\mathrm{H}$ : Adaptation of Aspergillus niger to several antifungal agents. Microbiology 1994, 140(9):2409-2414.

65. Hicks JK, Yu JH, Keller NP, Adams TH: Aspergillus sporulation and mycotoxin production both require inactivation of the FadA Ga protein-dependent signaling pathway. EMBO J 1997, 16(16):4916-4923. 
66. Jonsson P, Gullberg J, Nordström A, Kusano M, Kowalczyk M, Sjöström M, Moritz T: A strategy for identifying differences in large series of metabolomic samples analyzed by GC/MS. Anal Chem 2004, 76(6):1738-1745.

67. Jonsson P, Johansson Al, Gullberg J, Trygg J, Jiye A, Grung B, Marklund S, Sjöström M, Antti H, Moritz T: High-throughput data analysis for detecting and identifying differences between samples in GC/MS-based metabolomic analyses. Anal Chem 2005, 77(17):5635-5642.

doi:10.1186/1471-2180-12-106

Cite this article as: Yan et al:: Aspergillus flavus grown in peptone as the carbon source exhibits spore density- and peptone concentrationdependent aflatoxin biosynthesis. BMC Microbiology 2012 12:106.

\section{Submit your next manuscript to BioMed Central and take full advantage of:}

- Convenient online submission

- Thorough peer review

- No space constraints or color figure charges

- Immediate publication on acceptance

- Inclusion in PubMed, CAS, Scopus and Google Scholar

- Research which is freely available for redistribution 